\title{
Design Method of Aviation Architecture Based on Model Base
}

\author{
Zhijuan Zhan, Qing Zhou, Bingfei Li, and Bing Xue
}

\begin{abstract}
Drawing on dodaf ideas and summarizing aviation examples, the paper proposes an aviation architecture design method based on model base.The input content and form of aviation architecture design are defined, the organization form and metadata mapping relationship of meta model in the architecture model base are determined, the template data organization is designed. It realizes the automatic generation method of aviation system task architecture, functional architecture and requirements, and improves the efficiency of aviation architecture design.
\end{abstract}

Z. Zhan $(\bowtie) \cdot$ Q. Zhou $\cdot$ B. Li $\cdot$ B. Xue

China National Aeronautical Radio Electronics Research Institute, Shanghai 200233, China

e-mail: 13916905819@163.com

Q. Zhou

e-mail: Zhouqing0@163.com

B. $\mathrm{Li}$

e-mail: Libingfei612@126.com

B. Xue

e-mail: xueicb16@126.com

(C) The Author(s), under exclusive license to Springer Nature Switzerland AG 2021

D. Krob et al. (eds.), Complex Systems Design \& Management,

https://doi.org/10.1007/978-3-030-73539-5_41 